\title{
Glueballs and string breaking from full QCD*
}

SESAM and T $\chi$ L collaborations: G.S. Bali ${ }^{\mathrm{a}}$, N. Eicker ${ }^{\mathrm{b}}$, L. Giusti $^{\mathrm{c}}$, U. Glässner ${ }^{\mathrm{d}}$, S. Guesken ${ }^{\mathrm{d}}$,

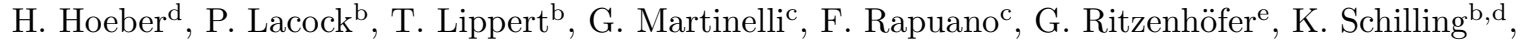
G. Siegert ${ }^{\mathrm{b}}$, A. Spitz ${ }^{\mathrm{b}}$, P. Ueberholz ${ }^{\mathrm{d}}$ and J. Viehoff ${ }^{\mathrm{d}}$

${ }^{a}$ Department of Physics, The University, Highfield, Southampton SO17 1BJ, UK

${ }^{b}$ HLRZ c/o Forschungszentrum Jülich, D-52425 Jülich and DESY, D-22603 Hamburg, Germany

${ }^{c}$ Dip. di Fisica, Univ. "La Sapienza" and INFN, Sezione di Roma, P'lle A. Moro, I-00185 Rome, Italy

${ }^{d}$ Physics Department, University of Wuppertal, D-42097 Wuppertal, Germany

${ }^{e}$ MIT, Center for Theoretical Physics, Cambridge, Massachusetts 02139, USA

We present results on the static potential, and torelon and glueball masses from simulations of QCD with two flavours of dynamical Wilson fermions on $16^{3} \times 32$ and $24^{3} \times 40$ lattices at $\beta=5.6$.

\section{SIMULATION}

The present simulations have been performed at various $\kappa$ values and lattice volumes at $\beta=5.6$ (Table 1). The effective lattice resolution ranges from $a^{-1} \approx 2.0 \mathrm{GeV}(\kappa=0.156)$ down to $a^{-1} \approx$ $2.5 \mathrm{GeV}(\kappa=0.158)$ while the ratio $m_{\pi} / m_{\rho}$ varies from 0.83 to 0.55 , corresponding to sea quarks that are slightly heavier than the strange quark and of about one quarter of its mass, respectively. In addition, results from quenched reference simulations at $\beta=6.0\left(a^{-1} \approx 2.1 \mathrm{GeV}\right)$ and $\beta=6.2$ $\left(a^{-1} \approx 3.1 \mathrm{GeV}\right)$ are presented.

Table 1

Simulation parameters.

\begin{tabular}{lrrrr}
\hline$\kappa$ & $V$ & $r_{0} a^{-1}$ & $n_{\text {glue }}$ & $n_{\text {pot }}$ \\
\hline 0.1560 & $16^{3} 32$ & $5.11(3)$ & 2129 & 236 \\
0.1565 & $16^{3} 32$ & $5.28(5)$ & - & 323 \\
0.1570 & $16^{3} 32$ & $5.46(5)$ & 2039 & 240 \\
0.1575 & $16^{3} 32$ & $5.98(7)$ & 2272 & 270 \\
0.1575 & $24^{3} 40$ & $5.93(4)$ & 1243 & 122 \\
0.1580 & $24^{3} 40$ & $6.33(7)$ & 743 & 95 \\
\hline$\beta=6.0$ & $16^{3} 32$ & $5.33(3)$ & - & 570 \\
$\beta=6.2$ & $32^{4}$ & $7.29(4)$ & - & 116 \\
\hline
\end{tabular}

On each of the small volumes, about 5000 thermalized trajectories have been generated. One

${ }^{*}$ Presented by Gunnar Bali. half of this amount has been achieved on the $24^{3} \times 40$ lattice at $\kappa=0.1575$ while the $\kappa=0.158$ run has not been completed yet. Glueball and torelon measurements $\left(n_{\text {glue }}\right)$ have been taken every 2 trajectories while smeared Wilson loops $\left(n_{\text {pot }}\right)$ were measured every 20 trajectories (16 at $\kappa=0.1565)$. Prior to statistical analysis, all data have been binned into blocks whose extent was large compared to the relevant autocorrelation time (see Ref. [1]).

\section{THE STATIC POTENTIAL}

An increase of the strength of the Coulomb-like attractive force between static sources at small separations, in respect to the quenched limit, has been observed previously [2]; the QCD coupling is running slower with the energy scale, once sea quarks are switched on. We confirm these findings with increased statistical accuracy and full control over systematic uncertainties (see Ref. [3]). Moreover, in extrapolating the Sommer scale $r_{0}^{-1}$ as a polynomial in the quark mass to the physical limit, we find a $\beta$ shift, $\beta_{n_{f}=0}-\beta_{n_{f}=2}=$ $0.57(2)$. This corresponds to an increase in the coupling of about $10 \%$ which is close to the naïve perturbative ratio $33 /\left(33-2 n_{f}\right)$.

With dynamical fermions, the static meson 
can decay into two static-light mesons. Ignoring meson-meson interactions, we expect the QCD string to "break" as soon as the potential exceeds twice the static-light mass, i.e. at about $1.5 \mathrm{fm}$. Neglecting quark mass effects on the dynamics of the binding problem (which is a reasonable assumption once this mass is small compared to a typical binding energy of $500 \mathrm{MeV}$ ), the string breaking distance should be shifted by an amount $\Delta r \approx 2 \Delta m / \sigma$ when changing the quark mass by $\Delta m . \quad \sigma=K a^{-2}$ denotes the effective string tension. From these considerations, we find $\Delta r<0.25 \mathrm{fm}$ for $\kappa \geq 0.156$. However, up to a separation of $2 \mathrm{fm}$ no indications of a flattening of the potential are found. We suspect that this is due to a bad overlap of the Wilson loop with the two-meson state.

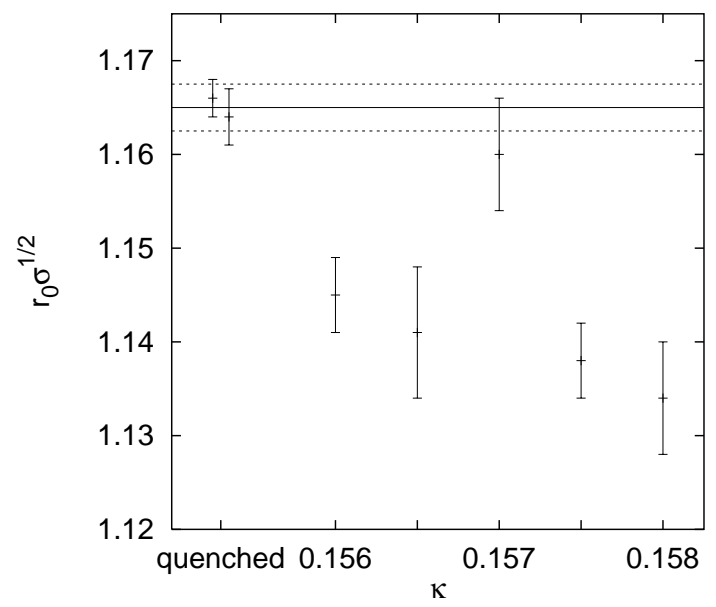

Figure 1. The effective string tension $\sigma$ in units of the Sommer scale $r_{0}$.

Reggè trajectories suggest values of 420 to $440 \mathrm{MeV}$ for the string tension. Fits of potential models to the bottomonium spectrum yield similar values. However, such parametrizations face difficulties in setting a reliable upper bound on $\sigma$; effects of an increased slope of the potential at large distance can be absorbed by decreasing the strength of the short range interaction. This is due to the fact that the spectrum is rather insensitive towards the shape of the potential outside of a region $0.2 \mathrm{fm}<r<1 \mathrm{fm}$. In contrast, the scale $r_{0}$ which is defined through the interquark force at intermediate distance can be constrained to $r_{0}^{-1} \approx 395 \mathrm{MeV}$ rather accurately. In Fig. 1, we display the value $r_{0} \sqrt{\sigma}$ against $\kappa$. While a quenched value $\sqrt{\sigma} \approx 460 \mathrm{MeV}$ appears to be reasonable, with two sea quarks this is reduced to about $450 \mathrm{MeV}$ such that for three active sea quarks a picture, consistent with the slope of Reggè trajectories, is likely to emerge.

\section{TORELON STATES}

Torelons are flux tubes encircling the periodic spatial lattice boundaries. We restrict ourselves to torelons with winding number one. As a consequence of the center group symmetry all such torelons are degenerate in the quenched case. Moreover, the mass of such a state is expected to equal $L_{S} a \sigma$ (up to tiny finite size corrections), where $L_{S} a$ denotes the spatial extent of the lattice.

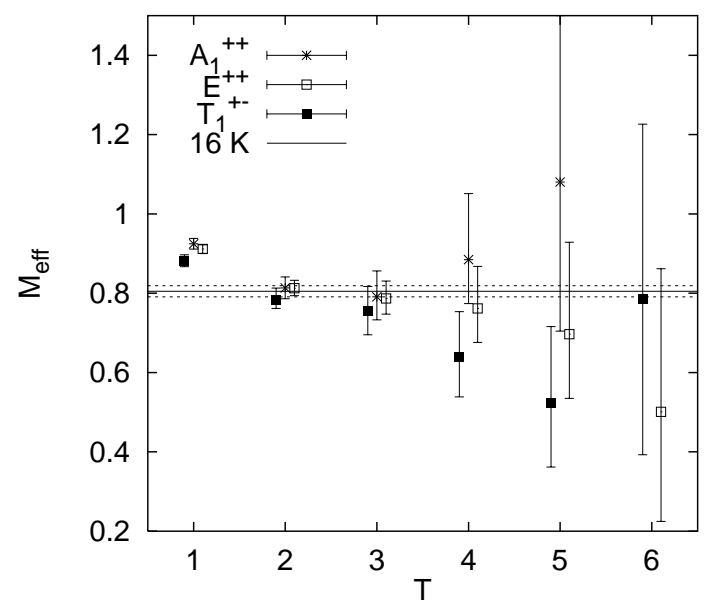

Figure 2. Effective torelon masses at $\kappa=0.156$.

With sea quarks, the $Z_{3}$ symmetry is only approximate and various torelon states, that can be classified in accord with irreducible representations of the cubic symmetry group, can obtain different masses. Moreover, mixing (or decays) between torelon states and isoscalar mesons might occur. The $T_{1}^{+-}$torelon is accompanied by the lightest isoscalar, the continuum $J=1 h_{1}(1190)$ meson.

In Fig. 2 2 we display effective masses of three different torelon states at $\kappa=0.156$. Note that all effective masses are strict upper limits on the real mass value. All states are degenerate and 


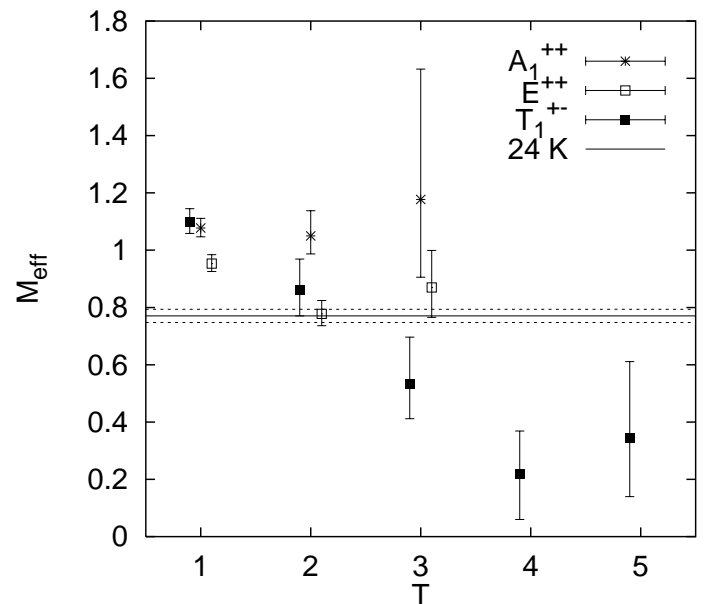

Figure 3. Effective torelon masses at $\kappa=0.158$.

in agreement with the expectation $L_{S} a \sigma$. The same holds true for $\kappa=0.157$. However, at $\kappa=0.1575$ and $\kappa=0.158$ we see indications of certain torelon states becoming lighter than expected. This situation is depicted in Fig. 3 for $\kappa=0.158$, where the $T_{1}^{+-}$state falls below $24 a \sigma$. Signals of the other states disappear into noise.

\section{GLUEBALLS}

At sufficiently light sea quark masses we expect the $0^{++}$glueball to mix with the two mesonic $I=0$ states of the $L=1$ nonet. Moreover, decays into two $\pi$ 's become possible. Results on the scalar $\left(A_{1}^{++}\right)$and tensor $\left(E^{++}\right)$glueballs are displayed in Table 2 and Fig. A. No indications of the mentioned effects are found.

Table 2

Glueball masses.

\begin{tabular}{lrrr}
\hline$\kappa$ & $L_{S}$ & $m_{0^{++}} r_{0}$ & $m_{2^{++}} / m_{0^{++}}$ \\
\hline 0.1560 & 16 & $3.56(12)$ & $1.62(07)$ \\
0.1570 & 16 & $3.01(18)$ & $1.89(13)$ \\
0.1575 & 16 & $3.26(25)$ & $1.86(13)$ \\
0.1575 & 24 & $4.27(23)$ & $1.46(28)$ \\
0.1580 & 24 & $4.49(41)$ & $1.54(21)$ \\
\hline$\beta=6.0$ & 20 & $3.69(23)$ & $1.67(15)$ \\
$\beta=\infty$ & - & $4.22(14)$ & $1.40(15)$ \\
\hline
\end{tabular}

In quenched simulations, finite size effects on the scalar glueball are small 㮌 as long as $m_{0^{++}}<$ $2 m_{t} \approx 2 L_{S} a \sigma$ where $m_{t}$ denotes the torelon

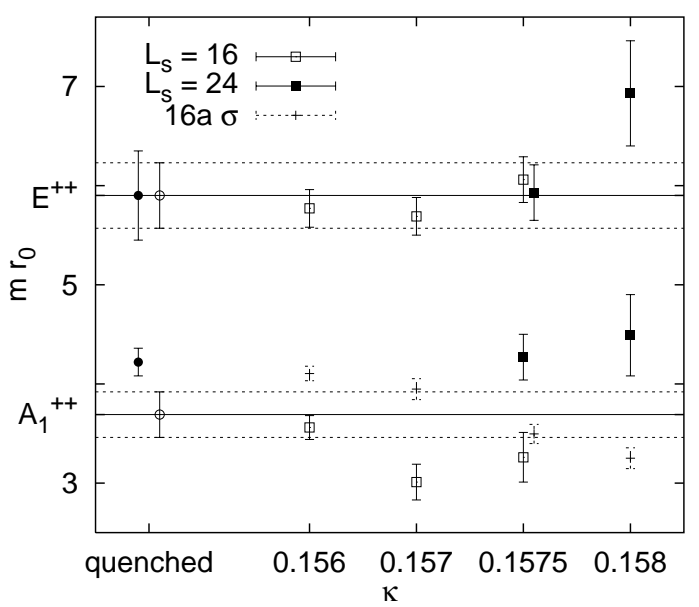

Figure 4. Glueball masses. Solid and open circles denote quenched results, extrapolated to the continuum, and at $\beta=6.0$, respectively.

mass. When including sea quarks, the glueball might break up and decay into a single torelon [5] through an intermediate mesonic state. The expected torelon masses $L_{S} a \sigma$ are included into Fig. A for $L_{S}=16$ (dashed error bars). Contrary to the quenched case, we indeed find significant FSE at $\kappa=0.1575$ (and probably $\kappa=0.157$ ). In general, the large volume data is consistent with quenched results. The $0^{++}$mass appears to exceed the $\beta=6.0$ reference value but agrees with the continuum extrapolated result.

\section{ACKNOWLEDGEMENTS}

We acknowledge support by the DFG (grants Schi 257/1-4 and Schi 257/3-2), the EU (contracts SC1*-CT91-0642, CHRX-CT92-0051 and CHBGCT94-0665) and PPARC (grant GR/K55738).

\section{REFERENCES}

1. $\mathrm{T} \chi \mathrm{L}$ collaboration: $\mathrm{T}$. Lippert et al., these proceedings.

2. SESAM collaboration: U. Glässner et al., Phys. Lett. B383 (1996) 98.

3. S. Guesken, these proceedings.

4. C. Michael and M. Teper, Nucl. Phys. B314 (1989) 347.

5. J. Kripfganz and C. Michael, Nucl. Phys. B314 (1989) 25. 